\title{
Neuroimmune Circuits Activated by Vagus Nerve Stimulation
}

\author{
Shuhei Kuwabara Eibhlin Goggins Shinji Tanaka \\ Division of Nephrology and Center for Immunity, Inflammation, and Regenerative Medicine, University of \\ Virginia, Charlottesville, VA, USA
}

\section{Keywords}

Acute kidney injury · Neuroimmune interactions · Spleen ·

Sympathetic nervous system · Vagus nerve

\begin{abstract}
The interaction between the nervous system and the immune system has recently been well-recognized. Vagus nerve stimulation (VNS) presents potential as an anti-inflammatory therapy through activation of neuroimmune pathways. Detailed understanding of the neuroimmune pathways VNS evokes is critical in order to successfully use it in the clinic for the treatment of acute kidney injury, in which inflammation plays an important role. In this review, we describe recent findings regarding VNS-induced neuroimmune pathways responsible for anti-inflammation and tissue protection.

(c) 2021 S. Karger AG, Basel
\end{abstract}

Contribution from the AKI and CRRT 2021 Symposium at the 26th International Conference on Advances in Critical Care Nephrology, A Virtual/Hybrid Event from San Diego, CA, USA, February 28 to March 5,2021 . This symposium was supported in part by the NIDDK funded University of Alabama at Birmingham-University of California San Diego O'Brien Center for Acute Kidney Injury Research (P30DK079337).

karger@karger.com

(c) 2021 S. Karger AG, Basel

www.karger.com/nef

Karger ${ }^{\prime}=$

\section{Introduction}

Inflammatory responses play a critical role in the maintenance of tissue homeostasis [1]. However, excessive and inappropriate activation of the immune system contributes to the pathogenesis and exacerbation of various inflammatory diseases. Recently, the interaction between the nervous system and the immune system has been recognized. Vagus nerve stimulation (VNS) has potential as an anti-inflammatory therapy by activating a neuroimmune circuit, the cholinergic anti-inflammatory pathway (CAP), which has been extensively reviewed [24]. In brief, the CAP is initiated by firing of the efferent vagus nerve. The signal is transmitted to the splenic nerve, resulting in the release of norepinephrine. Norepinephrine binds to $\beta_{2}$-adrenergic receptors expressed on a choline acetyltransferase-positive T-cell subpopulation in the spleen, which leads to the release of acetylcholine from these cells. Acetylcholine then binds to $\alpha_{7}$ nicotinic acetylcholine receptors ( $\alpha 7 \mathrm{nAChRs)}$ on macrophages, resulting in the suppression of pro-inflammatory cytokine production in the spleen. Thus, the canonical CAP involves the efferent vagus nerve, splenic nerve, $\beta_{2^{-}}$ adrenergic receptors on choline acetyltransferase-positive $\mathrm{T}$ cells, and $\alpha 7 \mathrm{nAChRs}$ on macrophages in the spleen. 


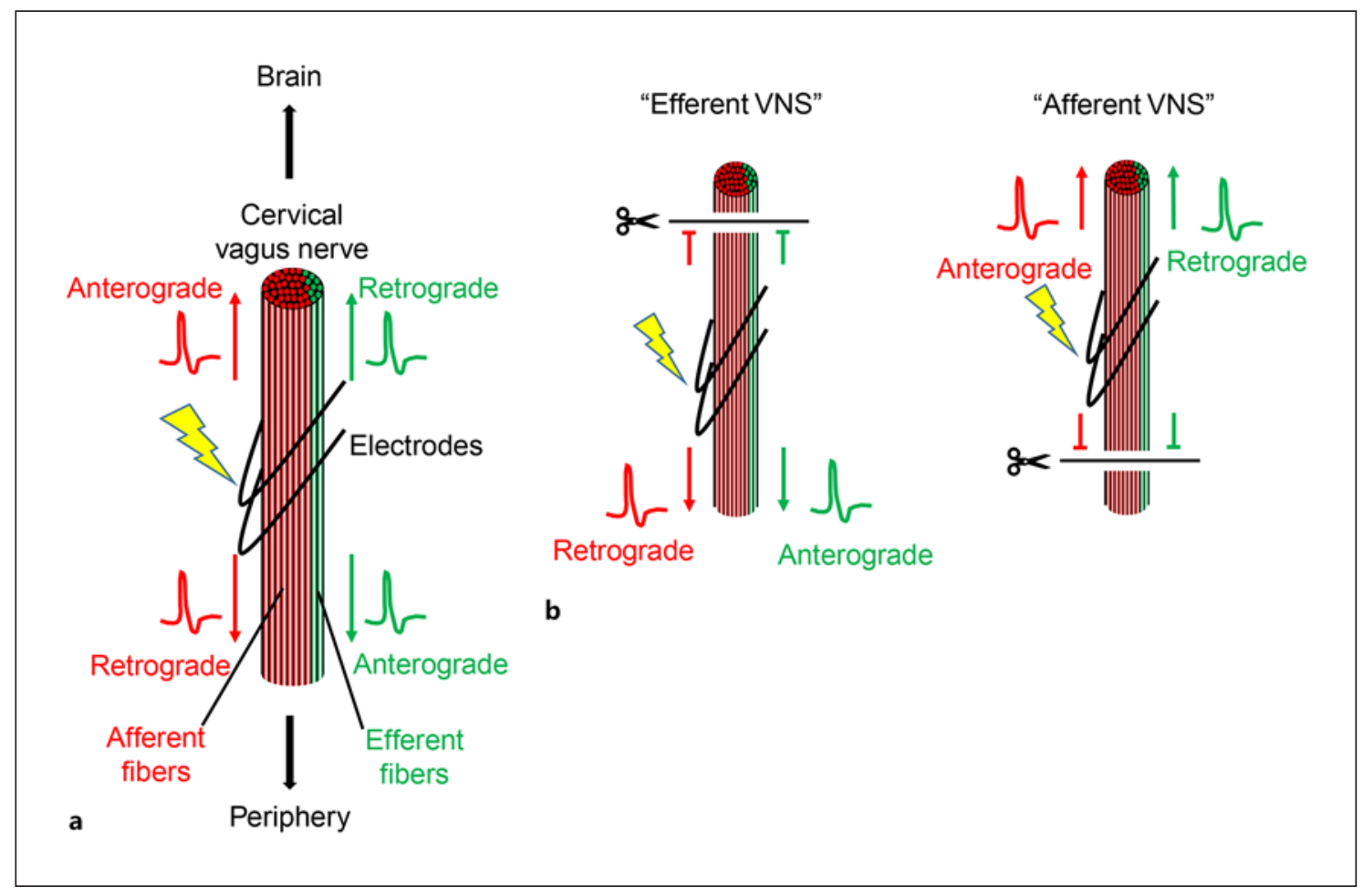

Fig. 1. Illustration depicting potential pathways in the cervical vagus activated by electrical stimulation. a Four distinct pathways in the cervical vagus are activated upon electrical VNS. Action potentials elicited by VNS are transmitted in 2 directions (anterograde and retrograde) in 2 types of fibers (efferent and afferent). $\mathbf{b}$ Two distinct pathways in the cervical vagus activated by "efferent VNS" and "afferent VNS." A local anesthetic can also be used to block nerve conduction. VNS, vagus nerve stimulation.

Recent studies have shown that VNS can also activate other neuroimmune pathways. In this review, we describe those recent findings, focusing on neural circuits responsible for anti-inflammation and tissue protection.

\section{Anti-Inflammatory Effects of Afferent VNS}

Electrical stimulation of the vagus (usually at the cervical vagus nerve trunk) activates not only efferent fibers but also afferent fibers. Indeed, the vagus nerve is composed of about $80 \%$ sensory afferent fibers and about $20 \%$ motor efferent fibers [5]. Emerging evidence suggests that afferent VNS also has an anti-inflammatory effect. In an arthritis model, afferent VNS activated the sympathetic nervous system through the central nervous system, which increased the release of norepinephrine from local sympathetic nerve terminals within inflamed joints to suppress the innate immune response [6]. The protective effect of afferent VNS was also observed in a lipopolysaccharide-induced endotoxemia model [7]; plasma levels of tumor necrosis factor were decreased by stimulation of abdominal vagal afferent fibers, and the anti-inflammatory effect was abolished when the splanchnic nerves were transected. These findings indicate that afferent VNS can elicit anti-inflammatory neuroimmune pathways that are distinct from the canonical CAP.

\section{Optogenetics: A Useful Tool to Dissect Neural Circuits}

Optogenetics enables us to manipulate the activity of neurons in a selective manner [8]. For example, channelrhodopsin-2 (ChR2), which is an excitatory light-sensitive opsin, is a nonselective cation channel; its gate is in open configuration only during blue light application. When blue light is applied to neurons that have been genetically modified to express ChR2, these neurons are depolarized via $\mathrm{Na}^{+}$entry. There are also inhibitory lightsensitive opsins that promote hyperpolarization of neurons. Halorhodopsin and archaerhodopsin function as an 


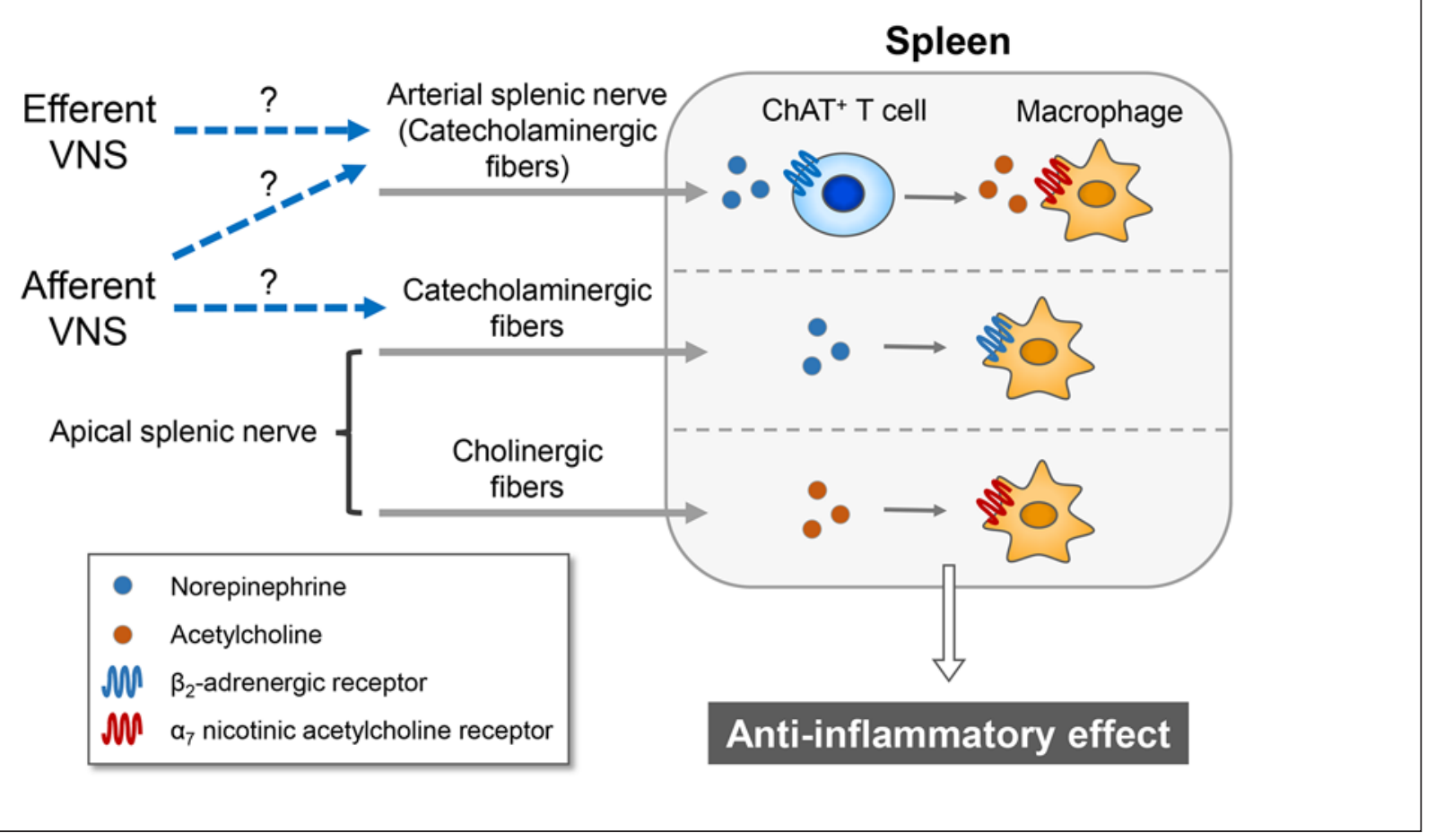

Fig. 2. Hypothetical neuroimmune interactions involving the splenic nerve branches in efferent/afferent VNS. Stimulation of the arterial splenic nerve, composed of catecholaminergic fibers, requires $\mathrm{T}$ cells to exert an anti-inflammatory effect. In contrast, stimulation of the apical splenic nerve, composed of both catecholaminergic and cholinergic fibers, does not require $\mathrm{T}$ cells but re-

inward $\mathrm{Cl}^{-}$pump and an outward $\mathrm{H}^{+}$pump when illuminated with yellow and green light, respectively. Thus, optogenetics is a powerful tool to selectively activate or inhibit neurons of interest and to dissect neural circuits responsible for the protective effect of VNS.

\section{Identifying Neural Circuits Responsible for VNS- Induced Kidney Protection}

In the mouse kidney ischemia-reperfusion injury (IRI) model, prior electrical stimulation of the cervical vagus nerve significantly ameliorated acute kidney injury and the kidney protection was mediated through $\alpha 7 \mathrm{nAChR}{ }^{+}$ splenocytes [9]. However, the precise neural circuit involved in kidney protection by VNS was unknown. When the vagus is electrically stimulated, action potentials are transmitted in 2 directions (anterograde and retrograde) in 2 types of fibers (efferent and afferent) (Fig. 1a). Thus, 4 distinct neural pathways can be activated by electrical quires both $\beta_{2}$-adrenergic receptors and $\alpha 7 n A C h R s$ on macrophages to exert an anti-inflammatory effect, with these 2 pathways acting in parallel. It remains unclear which component of the splenic nerve is activated by efferent/afferent VNS. VNS, vagus nerve stimulation.

VNS. Retrograde stimulation, especially in sensory afferent fibers, could contribute to anti-inflammatory and protective effects because it causes the release of various neuropeptides (such as substance $\mathrm{P}$ and calcitonin generelated peptide) at nerve terminals in thoracic and abdominal organs [10], and those peptides have immunomodulatory effects on immune cells $[11,12]$. However, due in part to prior methodological constraints, this concept had not yet been explored. In previous studies, "efferent VNS" or "afferent VNS" was performed by transecting or applying a local anesthetic to the vagus, in order to block nerve conduction prior to electrical VNS; however, this strategy still elicits a combination of anterograde and retrograde stimulation in efferent and afferent fibers (Fig. 1b).

Recently, we dissected out these 4 neural pathways in VNS by using optogenetics to identify neural circuits responsible for kidney protection [13]. We used ChatChR2 and Vglut2-ChR2 mice in which ChR2 is expressed in vagal efferents and vagal afferents, respectively. By 
combining selective stimulation of vagal efferents versus afferents with nerve conduction blockade, we demonstrated that anterograde efferent or afferent fiber stimulation was sufficient to protect the kidneys against IRI, while retrograde activation of either fiber type had no effect. We further identified the $\mathrm{C} 1$ neurons (in the medulla oblongata) - sympathetic nervous system - the splenic nerve - spleen - kidney axis as the downstream target of vagal afferent stimulation [13]. The importance of $\mathrm{C} 1$ neurons in kidney protection is consistent with a previous study in which optogenetic stimulation of $\mathrm{C} 1$ neurons was sufficient to protect the kidneys [14]. Adoptive transfer of splenocytes, but not lymph node cells or bone marrow cells, isolated from mice that underwent VNS protected recipient mice from renal IRI, which further supports a critical role of spleen/splenocytes in this context [13]. The signal from the splenic nerve seems to alter the phenotype of splenocytes, which promotes kidney protection; however, the mechanism for this has yet to be determined.

\section{Other Potential Neuroimmune Pathways Induced by VNS}

Recently, Guyot et al. [15] reported that there were 3 nerve branches projecting to the spleen in mice. Two of these ran in parallel with an artery and contained catecholaminergic fibers ("arterial splenic nerve"), whereas the other branch was located at the apex of the spleen and contained both catecholaminergic and cholinergic fibers ("apical splenic nerve") [15]. Arterial nerve stimulation required $\mathrm{T}$ cells to exert an anti-inflammatory effect, which is consistent with the canonical CAP. In contrast, an anti-inflammatory effect by apical nerve stimulation did not require $\mathrm{T}$ cells but required parallel activation of both $\beta_{2}$-adrenergic receptors and $\alpha 7 \mathrm{nAChRs}$ expressed on macrophages. It is unclear whether the cholinergic fibers in the apical nerve contain vagal preganglionic axons or are activated by vagal efferents. On the other hand,
Murray et al. [16] showed that an anti-inflammatory effect by efferent VNS required both T-cell-derived acetylcholine and $\beta_{2}$-adrenergic receptors, whereas afferent VNS required $\beta_{2}$-adrenergic receptors but not T cell-derived acetylcholine. Taken together, these findings may suggest that vagal efferent stimulation activates the arterial splenic nerve and requires T-cell-derived acetylcholine to exert an anti-inflammatory effect (canonical CAP), while vagal afferent stimulation activates catecholaminergic fibers in the apical splenic nerve (and arterial splenic nerve) and is independent from T cells (Fig. 2), which clearly warrants further investigation.

\section{Conclusion and Future Perspectives}

VNS has been explored as a promising strategy for the treatment of many inflammatory diseases because it activates the CAP. Notwithstanding, recent studies have revealed that VNS can also activate other anti-inflammatory neuroimmune pathways. One key question that remains in the kidney protection by VNS is how splenocytes with altered phenotypes after VNS protect the kidney. Precise understanding of the neuroimmune pathways evoked by VNS is critical for the successful clinical application of VNS, including noninvasive VNS [17], for the treatment of inflammatory diseases including acute kidney injury.

\section{Acknowledgements}

Research conducted for this publication was supported by the Uehara Memorial Foundation Research Fellowship and Japan Society for the Promotion of Science (JSPS) Overseas Research Fellowships (S.T.).

\section{Conflict of Interest Statement}

The authors report no conflicts of interest.

\section{References}

Neuroimmune Circuits Activated by

Vagus Nerve Stimulation
1 Rathinam VAK, Chan FK. Inflammasome, inflammation, and tissue homeostasis. Trends Mol Med. 2018;24:304-18.

2 Tanaka S, Okusa MD. Crosstalk between the nervous system and the kidney. Kidney Int. 2020;97:466-76.

3 Tanaka S, Okusa MD. Aki and the neuroimmune axis. Semin Nephrol. 2019;39(1):85-95.
4 Tanaka S, Hammond B, Rosin DL, Okusa MD. Neuroimmunomodulation of tissue injury and disease: an expanding view of the inflammatory reflex pathway. Bioelectron Med. 2019;5:13.

5 Foley JO, DuBois FS. Quantitative studies of the vagus nerve in the cat. I. The ratio of sensory to motor fibers. J Comp Neurol. 1937; 67(1):49-67. 
6 Bassi GS, Dias DPM, Franchin M, Talbot J, Reis DG, Menezes GB, et al. Modulation of experimental arthritis by vagal sensory and central brain stimulation. Brain Behav Immun. 2017;64:330-43.

7 Komegae EN, Farmer DGS, Brooks VL, McKinley MJ, McAllen RM, Martelli D. Vagal afferent activation suppresses systemic inflammation via the splanchnic anti-inflammatory pathway. Brain Behav Immun. 2018; 73:441-9.

8 Tanaka S, Okusa MD. Optogenetics in understanding mechanisms of acute kidney injury. Nephron. 2018;140:152-5.

9 Inoue T, Abe C, Sung SS, Moscalu S, Jankowski J, Huang L, et al. Vagus nerve stimulation mediates protection from kidney ischemiareperfusion injury through $\alpha 7 \mathrm{nAChR}+$ splenocytes. J Clin Invest. 2016;126:1939-52.
10 Chavan SS, Pavlov VA, Tracey KJ. Mechanisms and therapeutic relevance of neuro-immune communication. Immunity. 2017;46: 927-42.

11 Baral P, Umans BD, Li L, Wallrapp A, Bist M, Kirschbaum T, et al. Author Correction: Nociceptor sensory neurons suppress neutrophil and $\gamma \delta$ T cell responses in bacterial lung infections and lethal pneumonia. Nat Med. 2018; 24:1625-6.

12 Pinho-Ribeiro FA, Baddal B, Haarsma R, O'Seaghdha M, Yang NJ, Blake KJ, et al. Blocking neuronal signaling to immune cells treats streptococcal invasive infection. Cell. 2018;173:1083-97.e22.

13 Tanaka S, Abe C, Abbott SBG, Zheng S, Yamaoka Y, Lipsey JE, et al. Vagus nerve stimulation activates two distinct neuroimmune circuits converging in the spleen to protect mice from kidney injury. Proc Natl Acad Sci U S A. 2021;118(12):e2021758118.
14 Abe C, Inoue T, Inglis MA, Viar KE, Huang $\mathrm{L}$, Ye $\mathrm{H}$, et al. $\mathrm{C} 1$ neurons mediate a stressinduced anti-inflammatory reflex in mice. Nat Neurosci. 2017;20:700-7.

15 Guyot M, Simon T, Panzolini C, Ceppo F, Daoudlarian D, Murris E, et al. Apical splenic nerve electrical stimulation discloses an antiinflammatory pathway relying on adrenergic and nicotinic receptors in myeloid cells. Brain Behav Immun. 2019;80:238-46.

16 Murray K, Rude KM, Sladek J, Reardon C. Divergence of neuroimmune circuits activated by afferent and efferent vagal nerve stimulation in the regulation of inflammation. J Physiol. 2021;599(7):2075-84.

17 Addorisio ME, Imperato GH, de Vos AF, Forti S, Goldstein RS, Pavlov VA, et al. Investigational treatment of rheumatoid arthritis with a vibrotactile device applied to the external ear. Bioelectron Med. 2019;5:4. 